\title{
Erratum to: Choice and placebo expectation effects in the context of pain analgesia
}

\author{
Jason P. Rose • Andrew L. Geers • Heather M. Rasinski • \\ Stephanie L. Fowler
}

Published online: October 11, 2012

(c) Springer Science+Business Media New York 2012

\section{Erratum to: J Behav Med (2012) 35:462-470 \\ DOI 10.1007/s10865-011-9374-0}

The original version of this article unfortunately contained

errors in Table 1 . The corrected table is given below.

Table 1 Pain ratings as a function of choice condition

\begin{tabular}{|c|c|c|c|c|c|c|}
\hline \multirow[t]{2}{*}{ Measure } & \multicolumn{2}{|c|}{ Choice condition } & \multicolumn{2}{|c|}{ No choice condition } & \multicolumn{2}{|c|}{ Control condition } \\
\hline & M & SD & M & SD & M & SD \\
\hline \multicolumn{7}{|l|}{ Concurrent pain (s) } \\
\hline 15 & 2.05 & 1.49 & 3.38 & 2.17 & 2.57 & 1.93 \\
\hline 30 & 2.88 & 1.59 & 4.52 & 2.33 & 4.00 & 1.86 \\
\hline 45 & 3.65 & 2.66 & 6.09 & 2.43 & 5.65 & 1.68 \\
\hline 60 & 3.95 & 2.93 & 6.79 & 2.33 & 6.54 & 1.93 \\
\hline VAS post-task pain & 29.06 & 21.61 & 52.96 & 20.87 & 48.59 & 21.58 \\
\hline Anxiety & 2.98 & 1.56 & 4.56 & 2.03 & 4.54 & 1.78 \\
\hline
\end{tabular}

Concurrent pain ratings were made on an 11- point scale $(0=$ no pain; $10=$ worst possible pain $)$ at four time points during the task (after 15,30 , 45 , and $60 \mathrm{~s}$ ). VAS (visual analogue scale) post-task pain ratings were made by marking 100 -mm lines, anchored with no pain/not bad at all on the left side and worst pain possible/most unpleasant feeling possible on the right side. Anxiety ratings were made on a 7 -point scale $(1=n o t$ at all anxious; 7 = very anxious). The reported means control for participant age, gender, and baseline pain ratings

The online version of the original article can be found under doi:10.1007/s10865-011-9374-0.

J. P. Rose $(\bowtie) \cdot$ A. L. Geers · H. M. Rasinski .

S. L. Fowler

Department of Psychology, University of Toledo,

Mail Stop \#948, 2801 Bancroft St., Toledo,

OH 43606-3390, USA

e-mail: Jason.Rose4@utoledo.edu 